\title{
Impurity ion density measurements at the plasma edge of ASDEX Upgrade using lithium beam charge exchange spectroscopy
}

\author{
E. Wolfrum, J. Schweinzer, M. Reich, L.D.Horton, C.F. Maggi and the ASDEX Upgrade Team \\ Max-Planck-Institut für Plasmaphysik, EURATOM Association, Boltzmannstraße 2, D-85748 \\ Garching, Germany
}

\begin{abstract}
The method of lithium beam charge exchange spectroscopy is used to determine the density of $\mathrm{C}^{6+}$ and $\mathrm{He}^{2+}$ impurity ions at the edge of the ASDEX Upgrade tokamak plasma. Spectrally resolved measurements are used. l-level resolved atomic capture cross sections are corrected for collisional mixing. The absolute calibration relies on simultaneous beam emission spectroscopy. Together with detailed beam attenuation modelling, impurity ion densities can be measured with good accuracy.
\end{abstract}




\section{INTRODUCTION}

The H-mode regime is the choice for next step tokamak devices. It is characterised by the existence of a steep gradient region at the plasma edge, forming a so-called edge pedestal. Temperature values at the pedestal top determine the temperatures that can be reached in the plasma centre due to the self similarity of core temperature profiles ${ }^{1}$. As the physics in the pedestal region is not yet fully understood, a lot of effort has been devoted to the development of edge profile measurements with sufficient spatial resolution at ASDEX Upgrade ${ }^{2}$. By measuring the impurity ion density profiles, a better understanding of the processes which determine energy and particle transport in the pedestal region can be gained.

Along with edge Thomson scattering ${ }^{3}$ and multichannel FM reflectometry ${ }^{4}$, the Lithium beam is a tool particularly appropriate to probe the plasma edge ${ }^{5,6,7}$. At ASDEX Upgrade a fast 20-70 keV diagnostic Lithium beam is used to measure the electron density ${ }^{8}$ and the ion temperature ${ }^{9}$ at the plasma edge. While the electron density is determined from the emitted Li I (2s-2p) light, the ion temperature is derived from the line width of light emitted by impurity ions after charge exchange, i.e. electron capture from the Lithium beam donor atoms. With both installed Li-beam systems an accurate calibration of the charge exchange signal is achievable ${ }^{10}$. In this work we use the spectrally resolved charge exchange signal rather than the spectrally integrated signal. This is mandatory for the measurement of $\mathrm{He}^{2+}$ ion densities because of the large background signal from the cold $\mathrm{He}^{+}$ions. Moreover, we show that only careful handling of the necessary atomic data in a plasma environment yield accurate impurity ion density profiles in the outer pedestal region.

In the following we give a brief description of the experimental setup followed by a detailed explanation of the calibration procedure. Then the atomic data which were prepared for this work and are now implemented in the ADAS database are compared to experimental values where possible. The influence of the plasma environment on emission cross sections is demonstrated. Subsequently, all error sources are discussed and quantified and finally some results are shown for specific plasma discharges.

\section{EXPERIMENTAL SETUP}

The experimental setup of the neutral lithium beam diagnostics installed at ASDEX Upgrade has been described in detail elsewhere ${ }^{8,9}$. In the following, only a short description is given, to facilitate 
comprehension. The isotopically pure ${ }^{7} \mathrm{Li}$ ions are extracted from a $\beta$-eucryptite emitter, accelerated to the chosen energy and subsequently neutralised via charge exchange in a sodium vapour cell. In the measurements presented here, the Lithium beam energy was always set to $40 \mathrm{keV}$, optimising the charge exchange cross sections and neutralisation efficiency but compromising beam penetration into the plasma. With these settings the beam delivers a neutral beam equivalent current of about $2.2 \mathrm{~mA}$ within a diameter of $10-15 \mathrm{~mm}$. The beam is chopped by means of horizontal deflection plates positioned between ion source and neutraliser cell with a beam on:off cycle of 60:20 ms.

A schematic view of the position of the lithium beam and its optical systems is shown in figure $0^{11}$. The horizontal beam enters the plasma $32.6 \mathrm{~cm}$ above the tokamak midplane. The radial region of about $20 \mathrm{~cm}$ is observed by two optical systems: (i) The Li-IXS (Lithium beam impact excitation spectroscopy) system with a top down view images the region onto 35 fibres coupled to filter photomultiplier arrangements. The Li I (2s-2p) emission $(\lambda=670.8 \mathrm{~nm})$ is recorded with a spatial resolution of $5 \mathrm{~mm}$ and a temporal resolution of $200 \mu \mathrm{s}$. (ii) The Li-CXS optical system images the beam-plasma interaction region from below onto an array of 56 fibres, each covering a $5 \mathrm{~mm}$ diameter spot. Of these fibres 10 are chosen dependent on the actual plasma edge position and are connected to the $0.75 \mathrm{~m}$ Czerny-Turner spectrometer, equipped with a $1200 \mathrm{l} / \mathrm{mm}$ grating and a fast, back-illuminated frame-transfer CCD camera (Roper Scientific MicroMax: 512BFT). The camera was set up to record these 10 channels in frame-transfer mode with a temporal resolution of $7.8 \mathrm{~ms}$. In the course of this work, a second similar system was installed, so that 16 radial positions can be recorded simultaneously with the new setup. The number of radial channels per CCD was reduced and the temporal resolution increased to $4 \mathrm{~ms}$. The active charge exchange signal emitted in the small volume defined by the intersection of the respective line of sight and the Lithium beam is determined by subtraction of an averaged background of the temporally neighbouring 'beam off'-measurements from the 'beam on'-measurements. The data are subsequently fit with a Gaussian to determine the ion temperature.

Figure $2 \mathrm{a}$ and b show measured spectra of the C VI (7-8) charge exchange line at $529.2 \mathrm{~nm}$ and the He II (3-4) charge exchange line at $468.7 \mathrm{~nm}$, respectively. The data were taken in ELMy H-mode plasma discharges. While the errors of the measured spectra are equally distributed across the C VI line, there is a clear error maximum in the centre of the He II line due to the very large but spectrally narrow He II background radiation. In figure $2 \mathrm{~b}$ it can clearly be seen that in the region of the large error bars the data are not statistically distributed within the range of these error bars. This is due to the nature of the background, namely $\mathrm{He}^{+}$density fluctuations during or following ELMs. These 
fluctuations lead to strongly varying intensities of the cold He II background line and subsequently to the observed pixel-to-pixel correlated noise.

In this case the Gaussian fit to the error weighted data is dominantly determined by the wide wings yielding an accurate result for the otherwise undetermined line centre. Consequently the fitting parameters are used to calculate the spectrally integrated charge exchange signal $\mathrm{S}_{\mathrm{Z}}{ }^{\mathrm{CCD}}$.

\section{ABSOLUTE CALIBRATION}

This signal $\mathrm{S}_{\mathrm{Z}}{ }^{\mathrm{CCD}}$ is the locally emitted intensity multiplied by an absolute calibration factor $\mathrm{C}_{\mathrm{Z}}{ }^{\mathrm{CCD}}$. The intensity depends on the impurity ion density $\mathrm{n}_{\mathrm{Z}}$, the charge exchange emission rate $<\sigma \mathrm{v}_{\mathrm{Li}}>$ and the Lithium beam density $\mathrm{n}_{\mathrm{Li}}$. The signal can be written as

$$
\mathrm{S}_{\mathrm{Z}}^{\mathrm{CCD}}=\mathrm{C}_{\mathrm{Z}}^{\mathrm{CCD}} \mathrm{V}_{\mathrm{Li}} \mathrm{n}_{\mathrm{Z}} \mathrm{n}_{\mathrm{Li}} \sigma_{\lambda}{ }^{\text {eff }}
$$

where $\sigma_{\lambda}{ }^{\text {eff }}$ is the beam weighted effective emission cross section

$$
\sigma_{\lambda}{ }^{\text {eff }}=\Sigma_{n l} \mathrm{~N}_{\mathrm{Li}}(n l) \sigma_{\lambda}{ }^{\mathrm{eff}}(n l)
$$

Here $\mathrm{N}_{\mathrm{Li}}(\mathrm{nl})$ is the relative occupation number of $\mathrm{Li}(\mathrm{n} l)$ atoms in the beam, which is calculated by the beam attenuation code and is temporally and spatially dependant on the plasma electron density and temperature. $\sigma_{\lambda}{ }^{\text {eff }}(n l)$ denotes the effective emission cross section in a plasma environment after electron capture from a lithium atom in the state $\mathrm{nl}$ (see also next chapter).

Determination of the Lithium beam density and of the calibration factor is not necessary in this setup, because the Li I (2s-2p) emission is monitored on every plasma discharge with the Lithium beam IXS (impact excitation spectroscopy) system used to measure the edge electron density ${ }^{8}$. The signal $\mathrm{S}_{2 p}$ that is collected by a filter-photomultiplier arrangement can be written as $\mathrm{S}_{2 p}{ }^{\mathrm{PM}}=\mathrm{C}_{2 \mathrm{p}}{ }^{\mathrm{PM}} \mathrm{n}_{\mathrm{Li}} \mathrm{N}_{2 \mathrm{p}} \tau^{-1}$, with $\mathrm{C}_{2 \mathrm{p}}{ }^{\mathrm{PM}}$ the absolute calibration factor for this optical system, $\mathrm{n}_{\mathrm{Li}}$ the local particle density in the neutral beam, $\mathrm{N}_{2 p}$ the relative occupation of the $2 \mathrm{p}$ level and $1 / \tau$ the transition probability of the Li I (2s-2p) transition. 
For every spatial position of the charge exchange system the relation of the calibration factors $\mathrm{C}_{2 \mathrm{p}}{ }^{\mathrm{CCD}} / \mathrm{C}_{2 \mathrm{p}}{ }^{\mathrm{PM}}$ is determined by setting the spectrometer to the $\mathrm{Li}(2 \mathrm{~s}-2 \mathrm{p})$ transition line at $670.8 \mathrm{~nm}$ and comparing the signals $\mathrm{S}_{2 \mathrm{p}}{ }^{\mathrm{CCD}}$ and $\mathrm{S}_{2 \mathrm{p}}{ }^{\mathrm{PM}}$ :

$$
\mathrm{S}_{2 \mathrm{p}}^{\mathrm{PM}} / \mathrm{S}_{2 \mathrm{p}}{ }^{\mathrm{CCD}}=\mathrm{C}_{2 \mathrm{p}}{ }^{\mathrm{PM}} / \mathrm{C}_{2 \mathrm{p}}{ }^{\mathrm{CCD}} \text {. }
$$

The impurity ion density can now be derived from

$$
\mathrm{n}_{\mathrm{Z}}=\mathrm{S}_{\mathrm{Z}}{ }^{\mathrm{CCD}} / \mathrm{S}_{2 \mathrm{p}}{ }^{\mathrm{PM}} \mathrm{C}_{2 \mathrm{p}}{ }^{\mathrm{PM}} / \mathrm{C}_{2 \mathrm{p}}{ }^{\mathrm{CCD}} \mathrm{C}_{2 \mathrm{p}}{ }^{\mathrm{CCD}} / \mathrm{C}_{\mathrm{Z}}{ }^{\mathrm{CCD}} \mathrm{N}_{2 \mathrm{p}} /\left(\mathrm{v}_{\mathrm{Li}} \tau \sigma_{\lambda}{ }^{\text {eff }}\right) \text {. }
$$

Here the first term consists of the two measured signals on the CXS system and the IXS system, the second term is the measured relative response of the two systems to the Li I (2s-2p) transition, the third term is the relative response of the spectrometer system at the two different wavelengths which was accurately determined using an absolutely calibrated light source. The last term is composed of the constants $\mathrm{V}_{\mathrm{Li}}, \tau$ and $\sigma_{\lambda}{ }^{\text {eff }}$ as well as the relative occupation number of the $\operatorname{Li}(2 \mathrm{p})$ level, $\mathrm{N}_{2 \mathrm{p}}$. The latter can be determined in two ways: (i) it is routinely calculated during the evaluation procedure of the Li-IXS diagnostic, and (ii) it can be calculated in a forward calculation of the beam-attenuation code with the measured electron density and temperature profiles as input. For both methods the routinely used and well tested Lithium beam attenuation code is used ${ }^{12}$. It consists of a system of coupled differential equations for the occupation numbers $\mathrm{N}_{\mathrm{nl}}$ of the Lithium beam, taking into account excitation and de-excitation processes of $\mathrm{Li}$ atoms due to collisions with electrons, protons and impurities. Ionisation, charge exchange processes and radiative transitions are also included.

\section{ATOMIC DATA}

In order to determine the (n,l)-resolved charge exchange cross sections in collisions of $\mathrm{Li}$ atoms with $\mathrm{C}^{6+}$, calculations using the classical trajectory Monte Carlo (CTMC) method ${ }^{13}$ have been performed. This procedure involves numerically solving Hamilton's equations for a three-body system interacting solely via the Coulomb interaction. Numerous trajectories are integrated to emulate a mono-energetic beam of incident ions and to produce statistically reliable electron capture cross sections. A detailed description of the applied methods can be found in reference [14]. 
Atomic orbital close coupling calculations ${ }^{15}$ (AOCC) for $\mathrm{He}^{2+}$ colliding with Lithium atoms in various initial states $(2 \mathrm{~s}, 3 \mathrm{~s}, 2 \mathrm{p}, 3 \mathrm{p}, 3 \mathrm{~d})$ have been performed using a single set of basis states. The expansion includes travelling atomic orbitals on the $\mathrm{He}^{2+}$ centre (n-shells 1 - 6) and Li I states up to $\mathrm{Li}(5 \mathrm{~g})$. The latter states have been obtained by diagonalisation of the atomic Hamiltonian with a model potential. In addition to bound states pseudo-states on both centres are included which improve the convergence behaviour and represent ionisation. Altogether, the expansion consists of 87 states on the He centre and 49 on Li. From these calculations partial cross sections for charge exchange into He $\mathrm{II}(\mathrm{nl})(\mathrm{n}=1-6)$ have been derived in an energy range of 1.5 - $20 \mathrm{keV} / \mathrm{amu}$. The accuracy is $5-10 \%$ for cross sections $>10^{-15} \mathrm{~cm}^{2}$ and $30-40 \%$ for those $<10^{-16} \mathrm{~cm}^{2}$.

The determined state selective electron capture cross sections were written in the ADAS adf01 file format and thus made available for use with the ADAS program package ${ }^{16}$. The Atomic Data and Analysis Structure (ADAS) is a collection of codes and data for modelling the radiative properties of ions and electrons in plasmas (http://adas.phys.strath.ac.uk/). The code ADAS308 used in this work calculates effective emission cross sections taking into account all relevant effects occurring in a plasma environment (l-level mixing and cascade contributions applying branching ratios) for given relative velocities between $\mathrm{Li}$ atoms and impurity ions.

A comparison of the calculated emission cross sections without l-mixing (no plasma environment) with available measurements is shown in figure 0 . The data sets for the $\mathrm{He}^{2+}, \mathrm{C}^{6+}+\mathrm{Li}(2 \mathrm{~s})$ collisions measured at the Kernfysisch Versneller Instituut ${ }^{17},{ }^{18}$ are both consistently lower than the calculated values, the data measured by Aumayr et al. ${ }^{19}$ for $\mathrm{He}^{2+}+\mathrm{Li}(2 \mathrm{~s})$ agree very well with the calculated ones. The agreement between calculated and measured data for collisions with the $\mathrm{Li}(2 \mathrm{~s})$ ground state justifies a high confidence in the data for excited initial $\mathrm{Li}(\mathrm{n} l)$ states.

The importance of collisional mixing for the determination of effective emission cross sections was shown for charge exchange with deuterium neutral beams ${ }^{20}$. In the case of the Lithium beam edge diagnostic, it is even more important to take into account collisional mixing, because the plasma parameters change rapidly across the plasma edge. In figure 4 an example is given of the radial variation of the C VI effective emission cross section for discharge \#18055 at t = 3 - $5 \mathrm{~s}$. The electron density and temperature profiles (fig. 4a) show the characteristic steep gradients of an H-mode. In figure $4 \mathrm{~b}$ and $4 \mathrm{c}$ for $\mathrm{C}^{6+}$ and $\mathrm{He}^{2+}$, respectively, the effective emission cross sections $\sigma_{\lambda}{ }^{\text {eff }}(2 \mathrm{~s})$ and $\sigma_{\lambda}{ }^{\text {eff }}(2 p)$ as calculated by the ADAS routine for the given electron and temperature profiles are plotted versus a normalised radius ( $\rho_{\text {pol }}$ is normalised to poloidal flux, $\rho_{\text {pol }}=1$ indicates separatrix position). Also indicated (dotted lines) are emission cross sections for no l-mixing scenarios (upper 
boundary) as well as emission cross sections calculated under the assumption of complete collisional mixing (fully mixed, i.e. statistical population of states immediately after electron capture, lower boundary) for electron capture from $\mathrm{Li}(2 \mathrm{~s})$. It can clearly be seen that the correct treatment of $l$-level mixing in the cross sections has a strong impact on the determination of impurity ion densities. For the case of carbon, at $\rho_{\mathrm{pol}}=0.9$, the calculated emission cross sections are reduced as compared to the no l-mixing emission cross sections by factors 1.6 and 2.7 for electron capture from $\operatorname{Li}(2 \mathrm{~s})$ and $\mathrm{Li}(2 \mathrm{p})$, respectively. For Helium the reduction factors are somewhat smaller, 1.14 and 1.94, which is due to the fact that the upper level of the $\mathrm{C}$ VI line radiation consists of more l-levels $(n=8)$ than that of the He II transition $(n=4)$. Moreover, the radial change in emission cross section in the observed region has a direct influence on the measured impurity ion density gradient.

\section{ERRORS}

As the impurity ion densities are derived from a number of separate input data, the determination of an absolute error is not trivial. The main input data are: the CCD measurements of the charge exchange signal, the absolute calibration factors derived from cross calibration with the IXS system, the effective emission cross sections and the calculated Li-beam composition from the attenuation code. In the following we point out potential error sources and quantify them where possible.

As already shown in section II. the statistical errors of the averaged and background corrected data taken by the CCD are used as weights for the fitting routine (see figure 2). With the resulting fitting parameters the spectrally integrated charge exchange signal is calculated and with the corresponding errors of the fitting parameters a statistical error can be determined. For the spectra shown in figure 2 the signal is calculated to be $249+/-18$ (7\%) counts and $1559+/-162$ (10.4\%) counts for the C VI and the He II line, respectively, which are typical statistical errors of the measurements. In the case of helium, for plasma temperatures below $80 \mathrm{eV}$ the statistical errors from the Gauss fit become too large for a reasonable impurity ion density evaluation.

The relative calibration between IXS and CXS system is accurate to less than 4\%, but it must be ensured that there is no cross talk between CXS channels at all wavelengths used. Moreover, the beam position must be monitored carefully, as the upper optical system collects light from a wider spot normal to the beam direction than the lower system. To detect a relative shift in the horizontal

plane between the upper and the lower optical system, two fibres from the lower optical system are permanently connected to additional filter-photomultiplier detectors monitoring the $\mathrm{Li} \mathrm{I}(2 \mathrm{~s}-2 \mathrm{p}$ ) 
resonance line. If the monitored beam position is stable from one plasma discharge to the next, one reference measurement (spectrometer set to $\mathrm{Li}$ I at $670.8 \mathrm{~nm}$ ) per experimental day is sufficient.

Atomic data have estimated errors up to $10 \%$ for processes with a relevant contribution to the signal. Those charge exchange cross sections with larger errors are generally smaller than $10^{-16} \mathrm{~cm}^{2}$ and either occur in collisions of highly excited $\mathrm{Li}(\mathrm{n} l)$ or the electrons are captured into final n-states that are far away from the observed transition. In both cases the contribution to the measured signal and thus to the resulting error is very small $(<1 \%)$.

Treatment of collisional mixing has not been verified experimentally up to now, so that an error estimate cannot be given.

The assumption of just one collision energy rather than integrating over a Gauss distribution of the impurity ion energies is justified as long as the Li-beam energy is much higher than the impurity ion energy. With a maximum temperature of $1200 \mathrm{eV}$ in the observed plasma edge region the velocity of a $\mathrm{He}^{2+}$ ion is $0.3 \mathrm{keV} / \mathrm{amu}$ compared to $5.7 \mathrm{keV} / \mathrm{amu}$ for a $40 \mathrm{keV}$ Lithium beam. Even if the Li-beam energy is varied between 35 and $45 \mathrm{keV}$, the total effective emission cross section (weighted with the Li-beam composition) changes by less than $3 \%$ for given electron density and temperature profiles. Finally, the calculated composition and attenuation of the Li-beam is accurate to less than $5 \%$ for $n<$ 4, as could be demonstrated by the comparison of calculated and experimentally determined radial profiles of the most relevant excited states $\mathrm{Li}(2 \mathrm{p})$ and $\mathrm{Li}(3 \mathrm{~d})^{12}$.

Taking all errors into account (except from collisional mixing) a systematic error of $20 \%$ has to be added to the statistical errors from the measurements. The latter stems from the photon statistics of the charge exchange measurements and the subsequent fit to the measured data. The error variation depends mainly on the impurity ion density and the local current density of the Lithium beam.

\section{RESULTS IN EXAMPLES}

Figure 5 shows the $\mathrm{C}^{6+}$ density profile of discharge \#18055 as measured by the Lithium charge exchange system together with the edge portion of the profile measured with the core charge exchange system ${ }^{21}$. The plasma discharge is an H-mode with a plasma current of $0.8 \mathrm{MA}$, a central line averaged density of $5.510^{19} \mathrm{~m}^{-3}$, and heated with $5 \mathrm{MW}$ neutral beam injection. The plasma parameters were chosen to obtain an ELMy H-mode with low ELM frequency, so that the CCD camera frames which contain an ELM can be disregarded for the evaluation of the ion temperature. For the edge system, the $C^{6+}$ densities are evaluated with three different cross sections: no collisional mixing taken into account, full collisional mixing over the whole radial length and collisional mixing 
calculated with the ADAS package with the measured electron density and temperature profiles as input. Clearly, there is a large difference not only in the absolute values of the density but also in the resulting edge gradient, demonstrating the importance of a proper treatment of collisional mixing. The carbon concentration $\left(\mathrm{n}_{\mathrm{C} 6+} / \mathrm{n}_{\mathrm{e}}\right)$ is $0.7+/-0.1 \%$ at normalised radii $\rho_{\mathrm{pol}}=0.9-0.95$. In discharge \#18053, a similar H-mode discharge with a slightly higher central line averaged density of $6.310^{19}$ $\mathrm{m}^{-3}$, a He${ }^{2+}$ concentration $\left(\mathrm{n}_{\mathrm{He} 2+} / \mathrm{n}_{\mathrm{e}}\right.$ ) of $25+/-4 \%$ was measured at $\rho_{\mathrm{pol}}=0.9-0.95$.

The $\mathrm{He}^{2+}$ densities were also measured in ohmic discharges in ASDEX Upgrade. The selected discharge, only 27 plasma discharges ( $3^{\text {rd }}$ day) after wall boronisation, was carried out early in the morning, immediately after a 10 minute glow discharge in helium, a routine machine conditioning procedure at the beginning of an experimental day. A line integrated density of $3.810^{19} \mathrm{~m}^{-3}$ with no additional heating characterises the later part of the discharge, which is kept constant for two seconds. Figure 6 shows the electron and ion temperature profiles, the electron density profile, and the measured $\mathrm{He}^{2+}$ ion density for discharge \#20463 for the constant phase from 3.5 to $5.0 \mathrm{~s}$. In such low density discharges a typical concentration $\left(\mathrm{n}_{\mathrm{He} 2}+\mathrm{n}_{\mathrm{e}}\right.$ ) of around $12-15 \% \mathrm{He}^{2+}$ can be observed at a major radius between $\mathrm{R}=2.09$ and $2.13 \mathrm{~m}$ corresponding to a normalised poloidal radius $\rho_{\mathrm{pol}}$ of 0.91 and 0.99. In an earlier part from 1.7 to $2.3 \mathrm{~s}$ the discharge has an even lower line averaged density of $2.510^{19} \mathrm{~m}^{-3}$. Here the concentration rises to $20 \%$, indicating that the $\mathrm{He}^{2+}$ impurity ion density stays approximately constant for both density steps $\left(\mathrm{n}_{\mathrm{He} 2^{+}}=4-510^{18} \mathrm{~m}^{-3}\right)$.

\section{CONCLUSIONS}

The method of lithium beam charge exchange spectroscopy has been refined in this work, especially for the density measurement of $\mathrm{C}^{6+}$ and $\mathrm{He}^{2+}$ impurity ions at the plasma edge of the ASDEX Upgrade tokamak. Absolute calibration is achieved by simultaneous beam emission spectroscopy. The atomic data for electron capture from various initial Li states into $\mathrm{nl}$-resolved final states of $\mathrm{C}^{5+}$ and $\mathrm{He}^{+}$have been added to the ADAS data base.

For the correct determination of the impurity ion density gradient at the plasma edge it is important to include collisional mixing when calculating emission cross sections. The $\mathrm{C}^{6+}$ concentration $\left(\mathrm{n}_{\mathrm{C} 6+} / \mathrm{n}_{\mathrm{e}}\right)$ can be determined with high accuracy and is at present $<1 \%$. The quality of the $\mathrm{He}^{2+}$ ion density data is excellent in low density discharges, because fitting to the spectrally resolved data allows the determination of the line centre which is dominated by spectrally narrow He II background radiation. After boronisation, a He concentration $\left(\mathrm{n}_{\mathrm{He} 2}+\mathrm{n}_{\mathrm{e}}\right.$ ) between 12 and $20 \%$ is found in low density ohmic 
discharges. Impurity ion densities can be measured in H-modes in between ELMs by disregarding CCD camera frames which contain ELM induced signals.

\section{ACKNOWLEDGEMENTS}

We are grateful to Prof. Ron Olson for providing the CTMC calculations of $l$-resolved electron capture cross sections for the $\mathrm{Li}+\mathrm{C}^{6+}$ collision system in a fast and uncomplicated way. We also thank J. Neuhauser for his encouragement and critical comments. We would like to acknowledge that this work could not have been done without the contribution of the ASDEX Upgrade team as a whole.

\section{FIGURES}

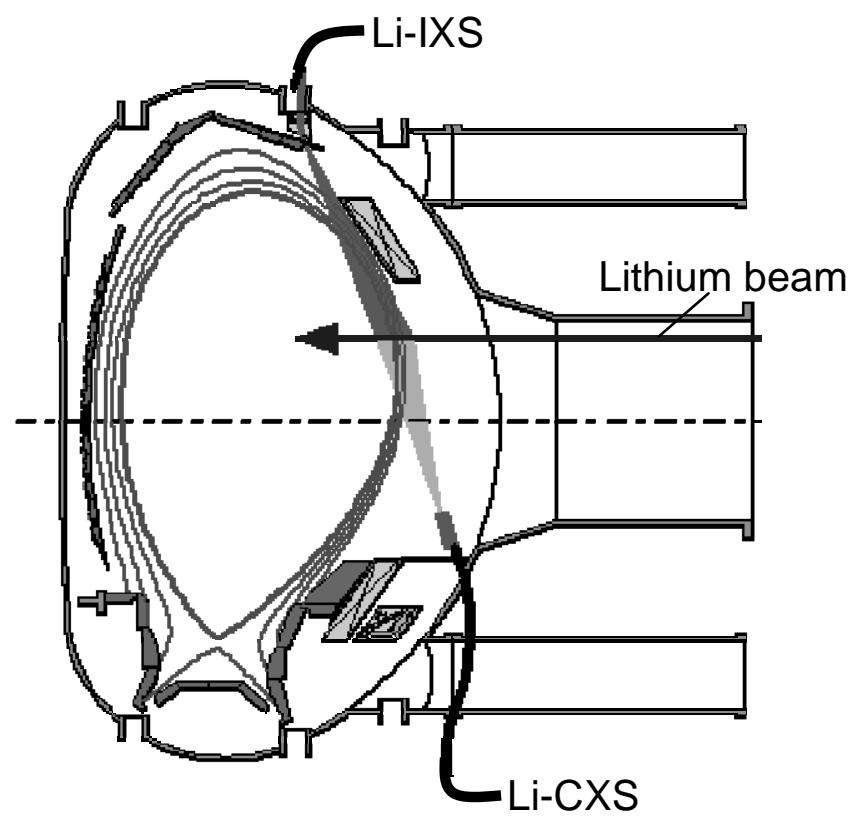

Figure 1: Toroidal cross section of ASDEX Upgrade with the position of the lithium beam and the two optical heads, one looking from above (Li-IXS) and the other from below (LiCXS) on the beam. 

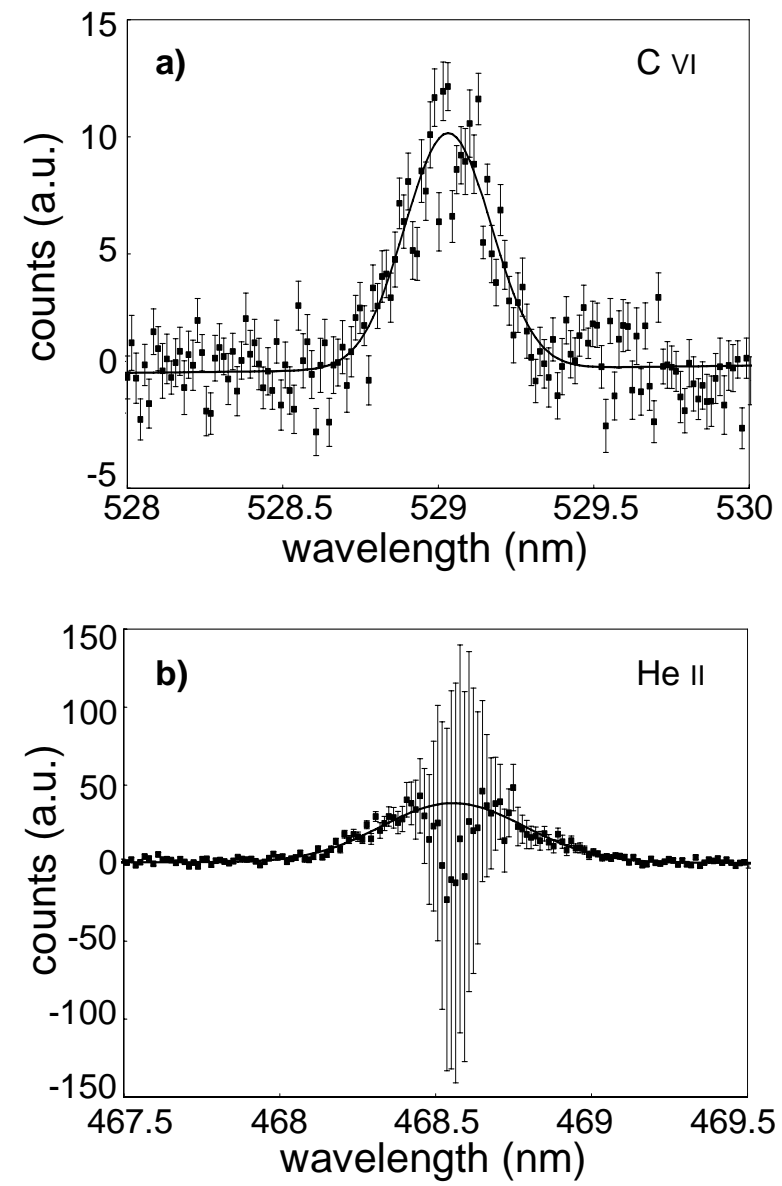

Figure 2: H-mode charge exchange spectra of a) carbon (\#18055, $t=3-5 s, T_{i}=770$ eV, $\rho_{\text {pol }}=$ 0.97) and b) helium (\#18053, t=3.5-4.2s, $\left.\mathrm{T}_{\mathrm{i}}=950 \mathrm{eV}, \rho_{\text {pol }}=0.97\right)$. Helium background radiation is much stronger due to ELMs and recycling from the vessel walls but restricted to a narrow spectral range. Thus, error bars (including correlated noise, see text) are large in the central wavelength region. C VI background radiation affects the whole line width. 


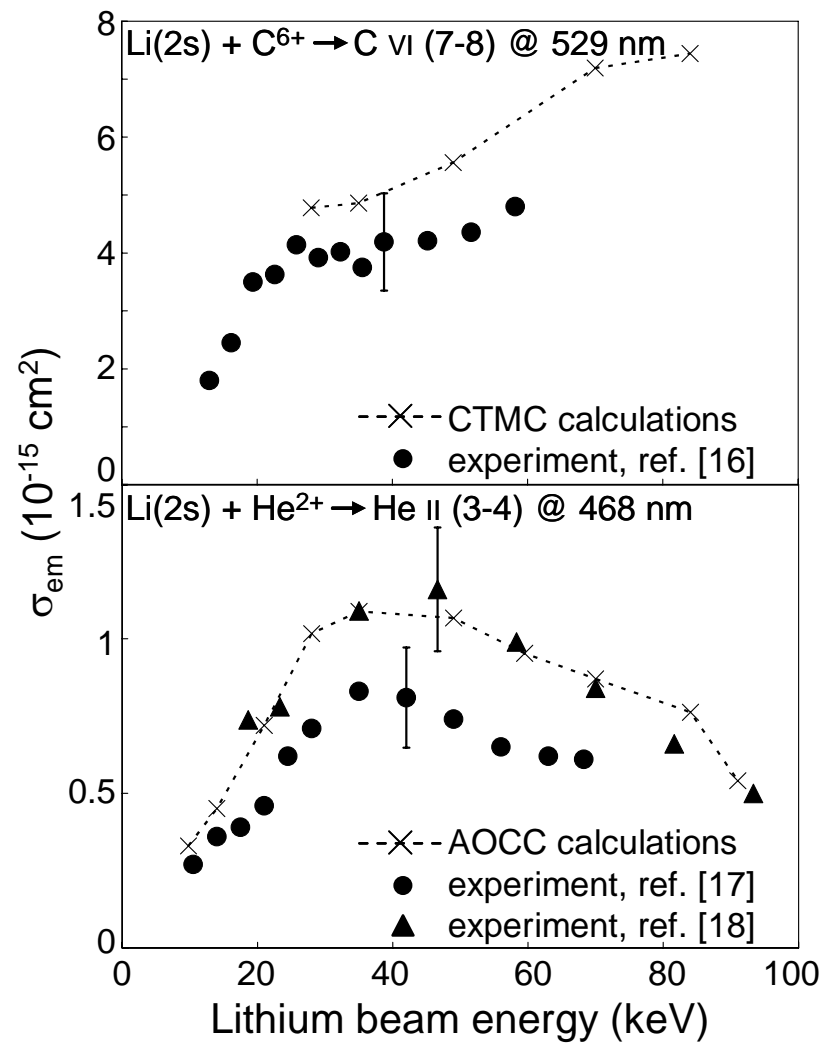

Figure 3: Comparison of calculated and measured emission cross sections for $\mathrm{Li}(2 \mathrm{~s})$ colliding with a) $\mathrm{C}^{6+}$ and b) $\mathrm{He}^{2+}$. 


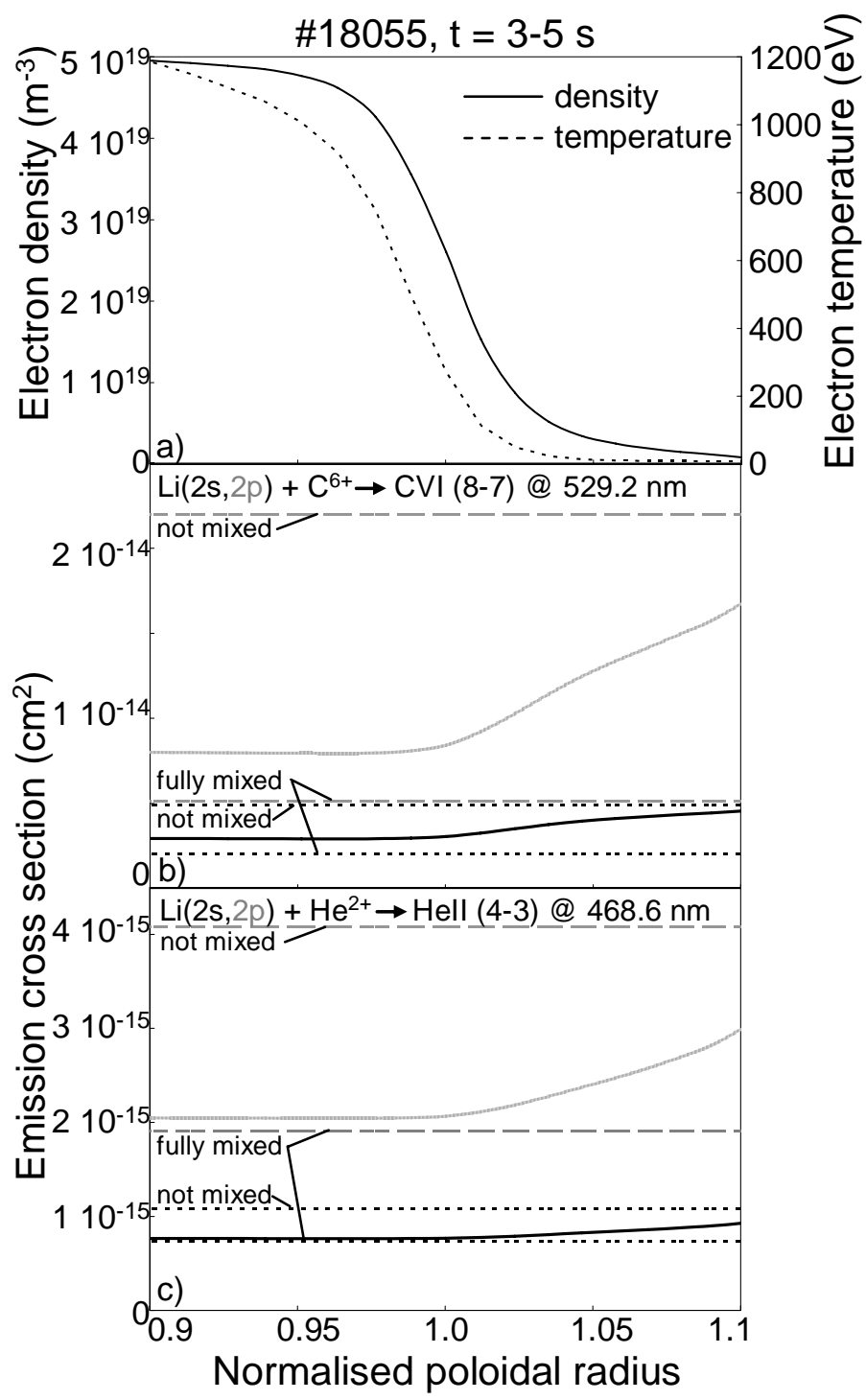

Figure 4: a) Electron density and temperature profiles for H-mode discharge \#18055 at t=3-5 s. These profiles are used to calculate emission cross sections for b) $C^{6+}$ at $529 \mathrm{~nm}$ and c) $\mathrm{He}^{2+}$ at $468 \mathrm{~nm}$. The black solid lines denote capture from the $\mathrm{Li}(2 \mathrm{~s})$ ground state, the grey solid lines denote capture from the $\mathrm{Li}(2 \mathrm{p})$ excited state. Also indicated are emission cross sections calculated with no collisional l-level mixing (upper boundary) as well as emission cross sections with full collisional l-level mixing (lower boundary). 


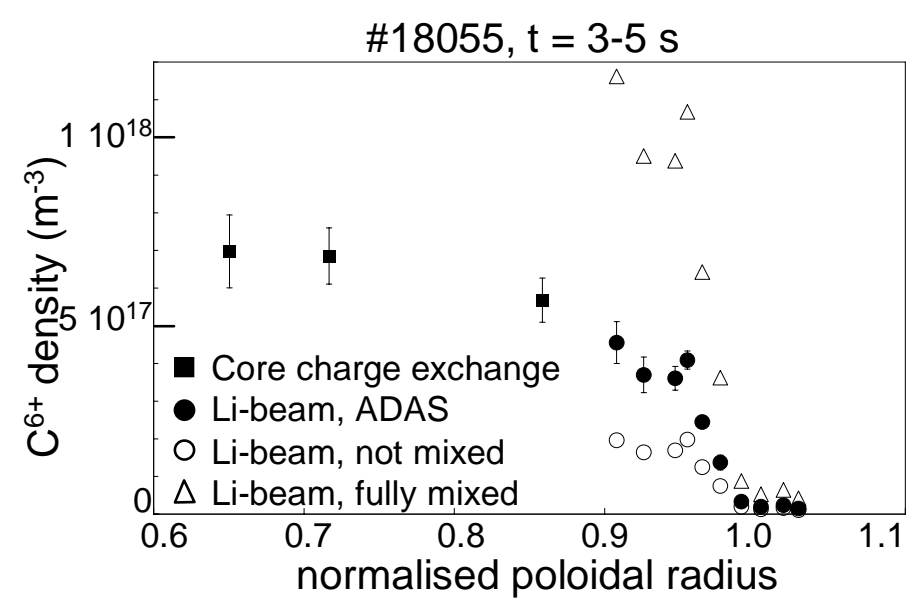

Figure 5: $\mathrm{C}^{6+}$ ion density profiles are shown for the H-mode discharge $\# 18055$ at $\mathrm{t}=3-5 \mathrm{~s}$. The full square symbols denote measurements from the core charge exchange system. The full circular symbols represent the Lithium beam measurements evaluated with emission cross sections calculated with the ADAS package and taking into account $l$ level mixing. The open symbols show the same measured data but evaluated without $l$ level mixing (circles) or full (i.e. statistical occupation) l-level mixing (triangles). 


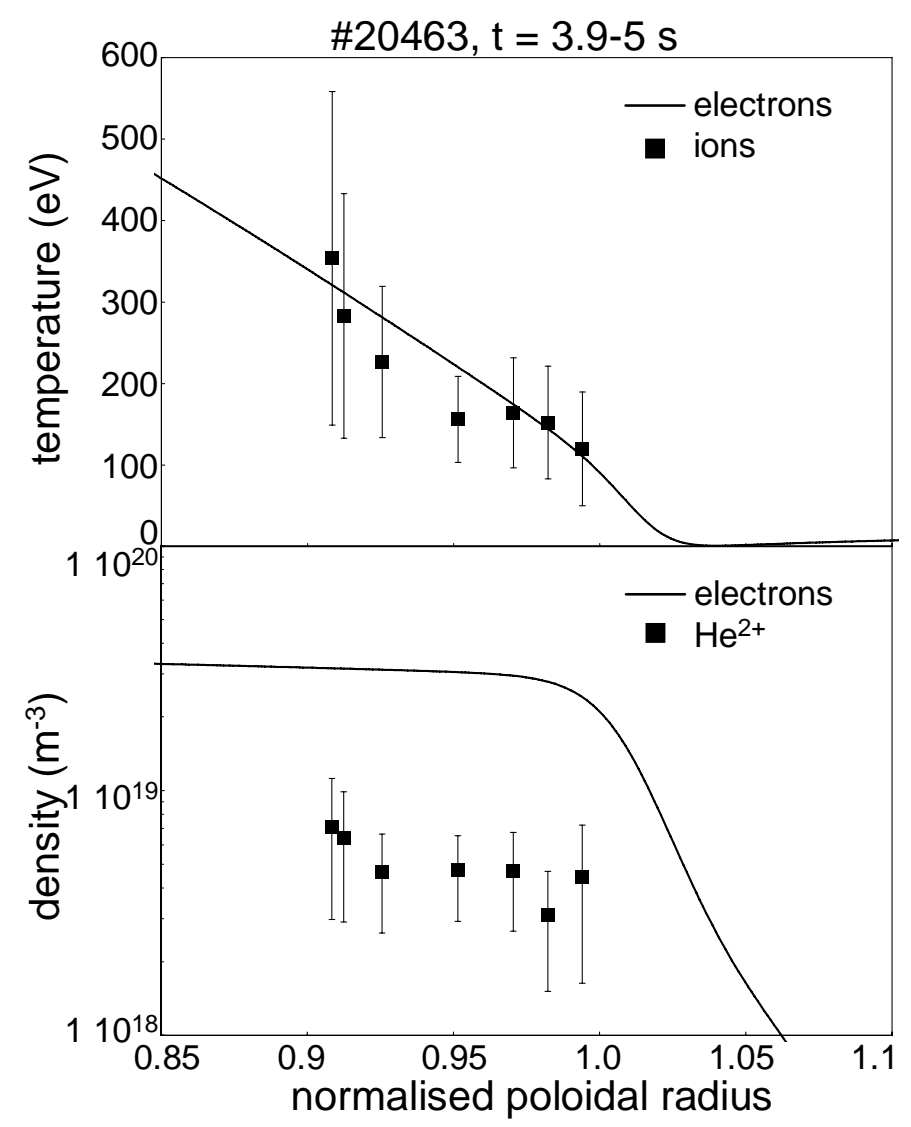

Figure 6: Electron and ion temperatures (upper graph) as well as electron and $\mathrm{He}^{2+}$ densities (lower graph) are shown for the standard ohmic discharge \#20463 averaged over the time period $\mathrm{t}=3.9-5 \mathrm{~s}$. 


\section{REFERENCES:}

1 F. Ryter, A. Staebler, G. Tardini, Fusion Science Technol. 44, 618-635 (2003)

2 L.D.Horton, A.V.Chankin, Y.P. Chen, G.D. Conway, D.P. Coster, T. Eich, E. Kaveeva, C. Konz, B. Kurzan, J. Neuhauser, I. Nunes, M. Reich, V. Rozhansky, S. Saarelma, J. Schirmer, J. Schweinzer, S. Voskoboynikov, E. Wolfrum and the ASDEX Upgrade Team, Nucl.Fusion 45, 856-862 (2005)

3 B. Kurzan, M. Jakobi, H. Murmann, Plasma Phys.Control.Fusion 46, 299-317 (2004)

4 C.Laviron, A.J.H. Donne, M.E. Manso and J. Sanchez, Plasma Phys. Control.Fusion 38, 905-936 (1996)

5 H. Ehmler, J. Baldzuhn, K. McCormick, A. Kreter, T. Klinger and W7-AS Team, Plasma Phys. Control. Fusion 45, 53-62 (2003)

6 E. Wolfrum, F. Aumayr, D. Wutte, HP. Winter, E. Hintz, D. Rusbueldt and R.P. Schorn, Rev. Sci. Instrum. 64, 2285-2292 (1993)

7 D.M.Thomas, A.W. Leonard and H.W. Mueller, Rev.Sci.Instr. 75, 4109-4111 (2004)

8 J. Schweinzer, E. Wolfrum, F. Aumayr, M. Poeckl, H. Winter, R.P. Schorn, E. Hintz and A. Unterreiter, Plasma Phys. Control. Fusion 34, 1173-1183 (1992)

9 M. Reich, E. Wolfrum, J. Schweinzer, H. Ehmler, L.D. Horton, J. Neuhauser and the ASDEX Upgrade Team, PLASMA Phys. Control. Fusion 46, 797-808 (2004)

10 R. Brandenburg, F. Aumayr, H. Winter, G. Petravich, S. Zoletnik, K. McCormick, J. Schweinzer and the W7-AS and ASDEX Upgrade Teams, Fusion Techn. 36, 289-295 (1999)

${ }^{11}$ M. Reich, Thesis, Ludwig Maximilian University, Munich (2005)

${ }_{12}$ R. Brandenburg, J. Schweinzer, S. Fiedler, F. Aumayr and HP. Winter, Plasma Phys. Control. Fusion 41, 471-484 (1999)

13 R.E. Olson and A. Salop, Phys. Rev. A16, 531-541 (1977)

${ }^{14}$ K.R. Cornelius, K. Wojtkowski, R.E. Olson, J.Phys.B:At.Mol.Opt.Phys. 33, 2017-2035 (2000)

15 J. Schweinzer, D. Wutte and HP. Winter, J.Phys.B: At.Mol.Opt.Phys. 27 (1994) 137-153

${ }^{16}$ H.P. Summers. Atomic data and analysis structure users manual, JET-IR (93) 07 (Abingdon: JET Joint Undertaking), 1993

17 E. Wolfrum, R. Hoekstra, F.J. DeHeer, R. Morgenstern, H. Winter, J.Phys.B: At.Mol.Opt.Phys. 25, 2597-2606 (1992)

${ }^{18}$ R. Hoekstra, E. Wolfrum, J.P.M. Beijers, F.J. DeHeer, H. Winter and R. Morgenstern, J.Phys.B: At.Mol.Opt.Phys. 25, 2587-2596 (1992) 
19 F. Aumayr, J. Schweinzer and H. Winter, J.Phys.B: At.Mol.Opt.Phys. 22, 1027-1034 (1989)

${ }^{20}$ H.P. Summers, H. Anderson, M.G. O’Mullane, M.G. von Hellermann, Physica Scripta T92, 80-84 (2001)

${ }^{21}$ C.F. Maggi, L.D. Horton, B. Zaniol, P. Franzen, H. Meister, D. Nishijuma, A. Staebler and ASDEX Upgrade Team, Proc. $30^{\text {th }}$ EPS Conf. on Controlled Fusion and Plasma Physics, Vol. 27A, P-1.63 (2003) 